This is the author's copy of the publication as archived with the DLR's electronic library at http://elib.dlr.de. Please consult the original publication for citation.

\title{
Collision Detection, Identification, and Localization on the DLR SARA Robot with Sensing Redundancy
}

Maged Iskandar, Oliver Eiberger, Alin Albu-Schäffer, Alessandro De Luca, and Alexander Dietrich

\section{Copyright Notice}

(C)2021 IEEE. Personal use of this material is permitted. However, permission to reprint/republish this material for advertising or promotional purposes or for creating new collective works for resale or redistribution to servers or lists, or to reuse any copyrighted component of this work in other works must be obtained from the IEEE.

\begin{tabular}{|c|}
\hline Citation Notice \\
\hline 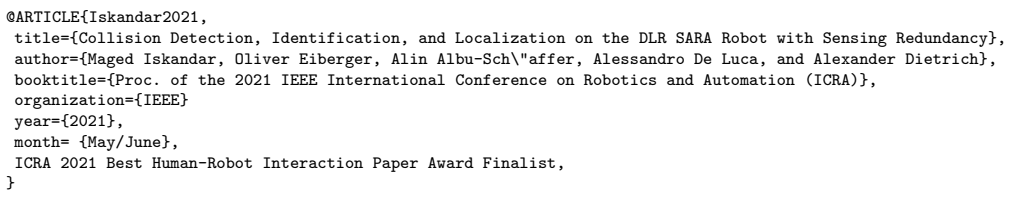 \\
\hline
\end{tabular}




\title{
Collision Detection, Identification, and Localization on the DLR SARA Robot with Sensing Redundancy
}

\author{
Maged Iskandar, Oliver Eiberger, Alin Albu-Schäffer, Alessandro De Luca, and Alexander Dietrich
}

\begin{abstract}
Physical human-robot interaction is known to be a crucial aspect in modern lightweight robotics. Herein, the estimation of external interactions is essential for the effective and safe collaboration. In this work, an extended momentumbased disturbance observer is presented which includes the sensing redundancy related to additional force-torque measurements. The observer eliminates the need for acceleration measurements/estimates and it is able to accurately reconstruct multiple simultaneous contact locations. Moreover, it provides uncoupled, configuration-independent, and singularity-free estimates of the external forces. The performance of the approach is experimentally validated on the SARA robot, the new generation of DLR lightweight robots, involving high resolution force-torque sensors in a redundant arrangement.
\end{abstract}

\section{INTRODUCTION}

Collaborative robots are increasingly becoming essential elements of the industry of the future, where they assist humans and cooperate with them hand-in-hand. Monitoring collisions is crucial to ensure safety at all times. One step towards human-robot collaboration involves the reliable handling of intentional interactions and collision scenarios. Detection, isolation, identification of collisions and ultimately building reaction strategies play a key role here [1].

Various model-based approaches have been developed in the context of collision detection and isolation [2]-[7]. The detection algorithm [2] uses a nonlinear adaptive impedance method, while [3] relies on a detailed actuator model to estimate joint-level disturbances. The work [4] modifies the idea of observing the joint velocity to estimate unknown external joint torques. Further, [7] employs the concept of monitoring the system momentum to provide dynamically uncoupled residual estimates and takes advantage of the integrated joint torque sensors. Most research works on detection and isolation of physical contacts focus on particular cases, for example, non-singular configurations [8], pure joint-level considerations [4], or a single collision/interaction point [9]. These restrictions are mainly due to limiting factors such as the sensitivity of the mapping between applied external forces and resulting joint external torques w.r.t. the joint configuration. Also, when the collision occurs on the links proximal to the base, the contact Jacobian is often rank deficient; thus, a unique mapping does not exist.

In many applications, when high fidelity contact information is required, the employment of additional force-torque sensors is the means of choice. A common distal location to attach such sensors is at the end-effector of the robot as the

A. De Luca is with the Sapienza Universita di Roma, Italy. The other authors are with the German Aerospace Center (DLR).

Contact: maged.iskandar@dlr.de

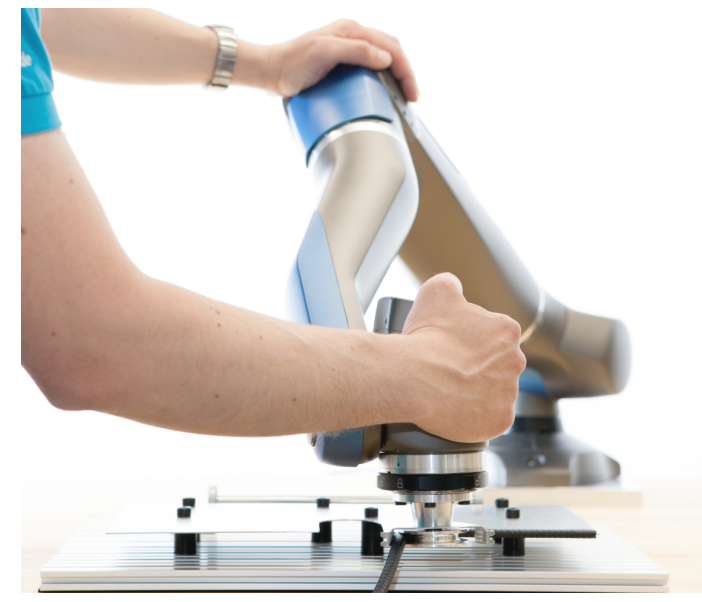

Fig. 1. Next generation of DLR lightweight robots: SARA (Safe Autonomous Robotic Assistant) in a typical collaborative task.

physical interaction is most likely occurring there [10], [11]. Also, such sensors could be included at the robot base [12][15] and/or even within the kinematic chain [16]. In order to acquire external interaction forces from such instrumentation, dynamical effects have to be treated correctly. This includes, among others, inertial and gravitational forces [17]. The challenge here is to precisely model and subsequently compensate for the dynamical effects that result from the robot motion. The Cartesian acceleration at the sensor location is required for this compensation, which can be obtained from the generalized (joint) accelerations. Practically speaking, that is a critical step since the acceleration is usually not measured but indirectly obtained from position signals. That, in turn, typically leads to high noise after numerical differentiation w.r.t. time. Several approaches exist for the acceleration estimation problem to be used to compensate for dynamical effects. In [9], [18] a method to estimate the joint acceleration separately using the momentum-based observer [7] is proposed. In the above-mentioned methods, the information from the additional force-torque sensors is not integrated into the estimation framework as a single step but it is treated separately as a parallel dynamic computation. Moreover, the obtained acceleration estimate is dynamically coupled through the inertia matrix, resulting in coupled dynamics for the estimated external forces/wrenches.

The contact localization problem has been addressed in [19] using model-based techniques and demonstrated by means of contacts at the end-effector of the manipulator with a simplified beam-like example. The literature also considers the employment of computer vision techniques [8], but also machine learning and optimization methods are used 
[20], [21]. The real-time estimation is still challenging and partially an open problem in the latter methods. However, with these methods the contact identification and localization is only possible near the end-effector and in non-singular configurations where the contact Jacobian has full rank.

In this work, the classical momentum-based disturbance observer [7] is extended to the case of redundancy in the force-torque measurements. We propose a unified framework to integrate all sensor signals and generate an extended residual. The approach eliminates the need for acceleration measurements or estimates and provides fully uncoupled dynamics for the obtained external forces and torques. Furthermore, multiple simultaneous contacts can be robustly identified and localized. Experiments on the DLR SARA robot with force-torque sensing redundancy, see Fig. 1, confirm the theoretical claims and demonstrate the advantages of the extended framework.

\section{FUndAMENTALS / BACKGROUND}

The rigid-body dynamics of a manipulator with $n$ degrees of freedom (DOF) can be written as

$$
\boldsymbol{M}(\boldsymbol{q}) \ddot{\boldsymbol{q}}+\boldsymbol{C}(\boldsymbol{q}, \dot{\boldsymbol{q}}) \dot{\boldsymbol{q}}+\boldsymbol{g}(\boldsymbol{q})=\boldsymbol{\tau}+\boldsymbol{\tau}^{\mathrm{ext}}
$$

with the generalized joint coordinates $\boldsymbol{q} \in \mathbb{R}^{n}$, the symmetric and positive definite inertia matrix $\boldsymbol{M}(\boldsymbol{q}) \in \mathbb{R}^{n \times n}$, the Coriolis and centrifugal matrix $\boldsymbol{C}(\boldsymbol{q}, \dot{\boldsymbol{q}}) \in \mathbb{R}^{n \times n}$, and the generalized gravity forces $\boldsymbol{g}(\boldsymbol{q}) \in \mathbb{R}^{n}$. Furthermore, the terms $\boldsymbol{\tau}, \boldsymbol{\tau}^{\text {ext }} \in \mathbb{R}^{n}$ describe the generalized joint forces and external forces, respectively. The external wrench $\boldsymbol{F}^{\text {ext }} \in \mathbb{R}^{m}$ is mapped to external joint torques through a geometric contact Jacobian matrix $\boldsymbol{J}_{c}(\boldsymbol{q}) \in \mathbb{R}^{m \times n}$ according to

$$
\boldsymbol{\tau}^{\mathrm{ext}}=\boldsymbol{J}_{c}(\boldsymbol{q})^{T} \boldsymbol{F}^{\mathrm{ext}}
$$

where $m=6$ if the full Cartesian space is considered. Typically the contact point/location and the associated $\boldsymbol{J}_{c}(\boldsymbol{q})$ are not exactly known. The joint-level external forces can be estimated by monitoring the generalized momentum [7] to avoid inversion of the inertia matrix [22]. This approach provides also decoupling for the estimated quantities, and eliminate the need for joint acceleration measurements. The momentum-based residual vector [7] can be defined as

$$
\boldsymbol{r}(t)=\boldsymbol{K}_{\mathrm{o}}\left(\boldsymbol{p}(t)-\int_{0}^{t}(\boldsymbol{\tau}-\boldsymbol{n}(\boldsymbol{q}, \dot{\boldsymbol{q}})+\boldsymbol{r}) d t-\boldsymbol{p}(\mathbf{0})\right),
$$

where $\boldsymbol{p}=\boldsymbol{M}(\boldsymbol{q}) \dot{\boldsymbol{q}}$ is the generalized momentum, the term $\boldsymbol{n}(\boldsymbol{q}, \dot{\boldsymbol{q}})=\boldsymbol{g}(\boldsymbol{q})+\boldsymbol{C}(\boldsymbol{q}, \dot{\boldsymbol{q}}) \dot{\boldsymbol{q}}-\dot{\boldsymbol{M}}(\boldsymbol{q}) \dot{\boldsymbol{q}}$ is introduced to provide a more compact expression, and $\boldsymbol{K}_{\mathrm{o}}$ is a diagonal gain matrix. The dynamics of the residual $\boldsymbol{r}$ is given by

$$
\dot{\boldsymbol{r}}=\boldsymbol{K}_{\mathrm{o}}\left(\boldsymbol{\tau}^{\mathrm{ext}}-\boldsymbol{r}\right), \quad r_{i}=\frac{1}{1+T_{i} s} \tau_{i}^{\mathrm{ext}}
$$

which can be regarded as a first-order filtered version of the actual external joint torque $\tau^{\text {ext }}$ following for $i=1 \ldots n$. Let $r_{i}$ and $\tau_{i}^{\text {ext }}$ be the joint-related components in the residual and external joint torques, respectively, expressed in the Laplace domain with Laplace variable $s$ and time constant $T_{i}=1 / K_{\mathrm{o}, i}$, which directly depends on the observer gain.
In the limit case when $K_{\mathrm{O}, i} \rightarrow \infty$ then $r_{i} \approx \tau_{i}^{\text {ext }}$. In practice, the observer gain is a trade-off between convergence rate of the residual and the noise level in the signal.

Generally, the residual is excited by collisions or interactions exerted in the task space and mapped to the joint space following (2). Assuming that $\boldsymbol{J}_{c}(\boldsymbol{q})$ is known, an estimate $\hat{\boldsymbol{F}}^{\text {ext }} \in \mathbb{R}^{m}$ of the external wrench can be obtained through

$$
\hat{\boldsymbol{F}}^{\mathrm{ext}}=\left(\boldsymbol{J}_{c}(\boldsymbol{q})^{T}\right)^{\#} \boldsymbol{r},
$$

where \# denotes a generalized pseudoinverse. The collision forces are often not fully reconstructable because of two main reasons. First, due to kinematic singularities when $\operatorname{rank}\left(\boldsymbol{J}_{c}(\boldsymbol{q})\right)<m$, meaning that there are less independent rows in $\boldsymbol{J}_{c}(\boldsymbol{q})$ than the dimension of the external force. In other words, $\boldsymbol{F}^{\text {ext }} \in \mathcal{N}\left(\boldsymbol{J}_{c}(\boldsymbol{q})^{T}\right)$ which means that at least one of the external force components is lying in the null space $\mathcal{N}$ of $\boldsymbol{J}_{c}(\boldsymbol{q})^{T}$ and counterbalanced by the mechanical structure of the robot [19]. The sensitivity for the estimation of the external wrench through (5) depends on the joint configuration, which limits the estimation to the components that can be properly detected by the residual, namely those inducing work on motion. The second scenario arises when the collision occurs below the $m$-th joint, so again $\operatorname{rank}\left(\boldsymbol{J}_{c}(\boldsymbol{q})\right)<m$. Intuitively speaking, the number of nonzero columns in $\boldsymbol{J}_{c}(\boldsymbol{q})$ is less than $m$. In other words, the residual of five joints or less is not sufficient to estimate the complete six-dimensional wrench. Moreover, if the complete wrench is not obtainable, even the contact localization is not possible in general. That poses the limitation that the contact point can only be uniquely determined beyond the sixth joint in case of full Cartesian space. That implies the necessary condition $i_{c} \geq 6$, where $i_{c} \in\{1 \ldots n\}$ denotes the index of the link in collision, and it is determined by the last non-zero component in the residual vector.

To improve the capability to estimate external disturbances, force-torque sensors are often added in various places in the structure of the robot, e.g., distal at the endeffector [23], at the robot base [12], or even within the kinematic chain [16]. One of the challenges here is to suppress/compensate correctly for dynamic effects and the load, in order to obtain the external disturbance. Generally, that requires acceleration measurements which are prone to noise or often not available in practice. It is indeed possible to estimate the generalized accelerations using the momentumbased observer but at the cost of nonlinear dynamic couplings among the joints. This effect can be observed when solving for the estimated generalized acceleration $\ddot{\hat{\boldsymbol{q}}} \in \mathbb{R}^{n}$ following

$$
\ddot{\hat{\boldsymbol{q}}}=\boldsymbol{M}(\boldsymbol{q})^{-1}(\boldsymbol{\tau}-\boldsymbol{C}(\boldsymbol{q}, \dot{\boldsymbol{q}}) \dot{\boldsymbol{q}}-\boldsymbol{g}(\boldsymbol{q})+\boldsymbol{r}) .
$$

The estimate is nonlinearly coupled due to the inversion of the inertia matrix. Based on (6) one can subsequently calculate the Cartesian acceleration at the location of any additional force-torque sensor (e.g. end-effector and base) and multiply by inertial parameters to compensate for dynamical effects. Nonetheless, such a compensation method leads to a dynamic coupling effect even if only one of the joints is 
subjected to a disturbance. In (1), dissipative friction effects can be included at different levels of complexity. However, in this work, we assume it is compensated by model-based and/or observer-based approaches [24]-[27]. Furthermore, the analysis conducted here will be restricted to the DLR lightweight robot SARA [28], which is designed with a fully integrated force-torque sensing redundancy and intended for highly dynamic trajectory tracking [29] and interaction control modes such as in [30]-[33]. The generalization to other types of robotic systems is straightforward.

\section{METHODS}

The residual information can be enriched by including sensing redundancy which potentially improves the capability of the estimation. We tackle this by introducing the concept of virtual joints to consider the physical presence of additional sensors. The basic concept is visualized in Fig. 2 with additional sensing devices (b for base, ui for user interface, ee for end-effector), which can be described by the dynamic equations of motions through

$$
\begin{aligned}
\overline{\boldsymbol{M}}(\overline{\boldsymbol{q}}) \ddot{\overline{\boldsymbol{q}}}+\overline{\boldsymbol{C}}(\overline{\boldsymbol{q}}, \dot{\overline{\boldsymbol{q}}}) \dot{\overline{\boldsymbol{q}}}+\overline{\boldsymbol{g}}(\overline{\boldsymbol{q}}) & =\boldsymbol{H} \boldsymbol{\tau}+\overline{\boldsymbol{\tau}}^{\mathrm{ext}}+\boldsymbol{A}(\overline{\boldsymbol{q}})^{T} \boldsymbol{\lambda} \\
\boldsymbol{A}(\overline{\boldsymbol{q}}) \dot{\overline{\boldsymbol{q}}} & =\mathbf{0}
\end{aligned}
$$

with $\overline{\boldsymbol{q}} \in \mathbb{R}^{\bar{n}}$ representing the generalized joint positions with $\bar{n}=n+k$ as the number of extended coordinates to account for the physical presence of additional sensors. These $k=n_{\mathrm{b}}+n_{\mathrm{ui}}+n_{\mathrm{ee}}$ DOF are considered as mechanically locked. The constraint Jacobian matrix imposed by the velocity constraints of the locked joints is $\boldsymbol{A}(\overline{\boldsymbol{q}}) \in \mathbb{R}^{k \times \bar{n}}$, and $\lambda \in \mathbb{R}^{k}$ describes the set of Lagrange multipliers. Let

$$
\overline{\boldsymbol{q}}=\left[\begin{array}{c}
\boldsymbol{q}_{\mathrm{b}} \\
\boldsymbol{q} \\
\boldsymbol{q}_{\mathrm{ui}} \\
\boldsymbol{q}_{\mathrm{ee}}
\end{array}\right], \quad \overline{\boldsymbol{\tau}}^{\mathrm{ext}}=\left[\begin{array}{c}
\boldsymbol{\tau}_{\mathrm{b}}^{\mathrm{ext}} \\
\boldsymbol{\tau}_{\mathrm{ext}}^{\mathrm{ext}} \\
\boldsymbol{\tau}_{\mathrm{ui}}^{\mathrm{ext}} \\
\boldsymbol{\tau}_{\mathrm{ee}}^{\mathrm{ext}}
\end{array}\right]
$$

which contains the additional coordinates $\boldsymbol{q}_{\mathrm{b}} \in \mathbb{R}^{n_{\mathrm{b}}}$ for a base sensor, $\boldsymbol{q}_{\mathrm{ui}} \in \mathbb{R}^{n_{\mathrm{ui}}}$ for a user-interface sensor, and $\boldsymbol{q}_{\mathrm{ee}} \in$ $\mathbb{R}^{n_{\text {ee }}}$ for an end-effector sensor. Such sensor devices can have various complexity in terms of the number of directions to measure, but the most common type is certainly the classical six-dimensional force-torque sensor. Therefore, the physical presence of such instrumentation could be regarded as a mechanically locked six-dimensional (6DOF) joint. Accordingly, the generalized external forces are augmented to $\bar{\tau}^{\text {ext }} \in \mathbb{R}^{\bar{n}}$. The virtual joints shown in Fig. 2 are kept due to the measured signals, but they are considered as constrained/non-moving joints in the augmented dynamics. Moreover, it is constrained kinematically so that (8) holds true, and $\boldsymbol{A}(\overline{\boldsymbol{q}})^{T} \boldsymbol{\lambda}$ are the joint forces and torques acting against the constraints. The term $\boldsymbol{H} \in \mathbb{R}^{\bar{n} \times n}$ describes the mapping from actuation forces to generalized forces acting collocated to $\overline{\boldsymbol{q}}$. The overall inertia matrix $\overline{\boldsymbol{M}}(\overline{\boldsymbol{q}}) \in \mathbb{R}^{\bar{n} \times \bar{n}}$, the Coriolis and centrifugal matrix $\overline{\boldsymbol{C}}(\overline{\boldsymbol{q}}, \dot{\overline{\boldsymbol{q}}}) \in \mathbb{R}^{\bar{n} \times \bar{n}}$, and the generalized gravity forces $\overline{\boldsymbol{g}}(\overline{\boldsymbol{q}}) \in \mathbb{R}^{\bar{n}}$ in (7) are extended analogously. The dynamic structure of (7) and (8) can be used to express the system when encapsulating the constrained joints using six-dimensional force-torque sensors.

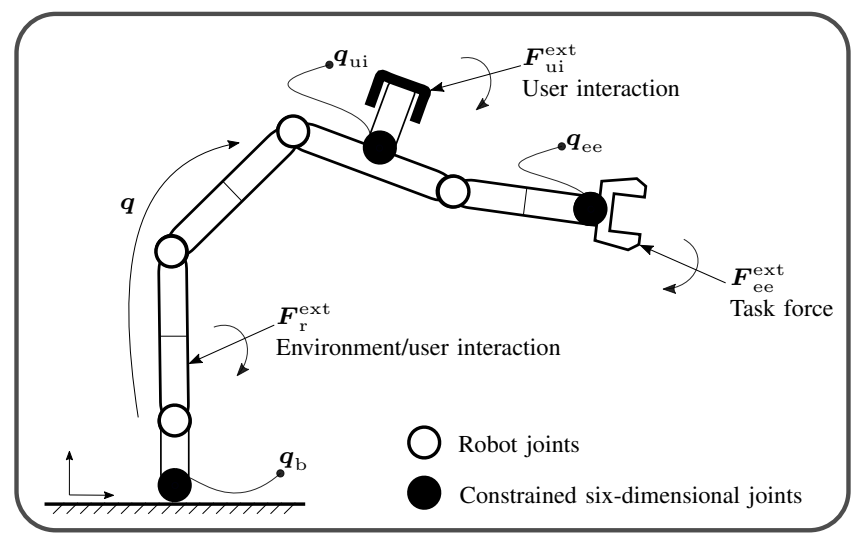

Fig. 2. The concept of including the sensing redundancy assuming extra states (DOF) for the sensors can be visualized as virtual constrained sixdimensional joints. The black circles indicate a possible arrangement of six-dimensional force-torque sensors, which is regarded here as sensorized constrained joints. That demonstrates a typical example for a robotic manipulator with fully integrated force-torque sensing redundancy.

The graphical illustration of this concept is shown in Fig. 2, where the constrained sensorized joints (black) represent the force and torque sensing capability at specific points in the articulated chain. Now all dynamic coupling effects of the multi-body system are already included in the model. One can define the external wrench at the locations b, ui, and ee:

$$
\boldsymbol{\tau}_{\mathrm{b}}^{\text {ext }}=\left[\begin{array}{c}
\boldsymbol{f}_{\mathrm{b}}^{\text {ext }} \\
\boldsymbol{m}_{\mathrm{b}}^{\text {ext }}
\end{array}\right], \boldsymbol{\tau}_{\mathrm{ui}}^{\text {ext }}=\left[\begin{array}{c}
\boldsymbol{f}_{\mathrm{ui}}^{\text {ext }} \\
\boldsymbol{m}_{\mathrm{ui}}^{\text {ext }}
\end{array}\right], \boldsymbol{\tau}_{\mathrm{ee}}^{\text {ext }}=\left[\begin{array}{c}
\boldsymbol{f}_{\mathrm{ee}}^{\text {ext }} \\
\boldsymbol{m}_{\mathrm{ee}}^{\text {ext }}
\end{array}\right]
$$

where $\boldsymbol{f}_{\mathrm{b}}^{\text {ext }}, \boldsymbol{f}_{\mathrm{ui}}^{\text {ext }}, \boldsymbol{f}_{\mathrm{ee}}^{\text {ext }} \in \mathbb{R}^{3}$ and $\boldsymbol{m}_{\mathrm{b}}^{\text {ext }}, \boldsymbol{m}_{\mathrm{ui}}^{\text {ext }}, \boldsymbol{m}_{\mathrm{ee}}^{\text {ext }} \in \mathbb{R}^{3}$ represent the force and torque components exerted at the base, user interface, and the end-effector, respectively. The extended generalized momentum of the robot including the virtual joints (additional sensors) is defined as

$$
\overline{\boldsymbol{p}}=\overline{\boldsymbol{M}}(\overline{\boldsymbol{q}}) \dot{\bar{q}}
$$

resulting in an observer dynamics of the form

$$
\begin{aligned}
\dot{\overline{\boldsymbol{p}}} & =\overline{\boldsymbol{\tau}}-\overline{\boldsymbol{n}}(\overline{\boldsymbol{q}}, \dot{\overline{\boldsymbol{q}}})+\overline{\boldsymbol{r}}(t) \\
\dot{\overline{\boldsymbol{r}}} & =\overline{\boldsymbol{K}}_{\mathrm{O}}(\dot{\overline{\boldsymbol{p}}}-\dot{\overline{\hat{\boldsymbol{p}}}})
\end{aligned}
$$

One can define the augmented sensed quantities used in the observer dynamics/equations as

$$
\bar{\tau}=\left[\begin{array}{c}
\tau_{\mathrm{b}} \\
\tau \\
\tau_{\mathrm{ui}} \\
\tau_{\mathrm{ee}}
\end{array}\right]
$$

The variables $\tau_{\mathrm{b}}, \tau_{\text {ui }}$, and $\tau_{\mathrm{ee}}$ represent the base, userinterface, and end-effector measurements, and $\tau$ is the covector of joint torques. The extended residual vector $\overline{\boldsymbol{r}}(t)$ (observer output) can be obtained in a straightforward way by substituting (12) in (13) and integrating it w.r.t. time as

$$
\overline{\boldsymbol{r}}(t)=\overline{\boldsymbol{K}}_{\mathrm{O}}\left(\overline{\boldsymbol{p}}(t)-\int_{0}^{t}(\overline{\boldsymbol{\tau}}-\overline{\boldsymbol{n}}(\overline{\boldsymbol{q}}, \dot{\overline{\boldsymbol{q}}})+\overline{\boldsymbol{r}}) d t-\overline{\boldsymbol{p}}(0)\right)
$$

with the diagonal gain matrix $\overline{\boldsymbol{K}}_{o}=\operatorname{diag}\left(\bar{K}_{o, 1}, \ldots, \bar{K}_{o, \bar{n}}\right)$ of the observer, and $\overline{\boldsymbol{n}}=\overline{\boldsymbol{g}}+\overline{\boldsymbol{C}} \dot{\overline{\boldsymbol{q}}}-\dot{\overline{\boldsymbol{M}}} \dot{\overline{\boldsymbol{q}}}$. Note that the dependencies on the states have been omitted for the sake 


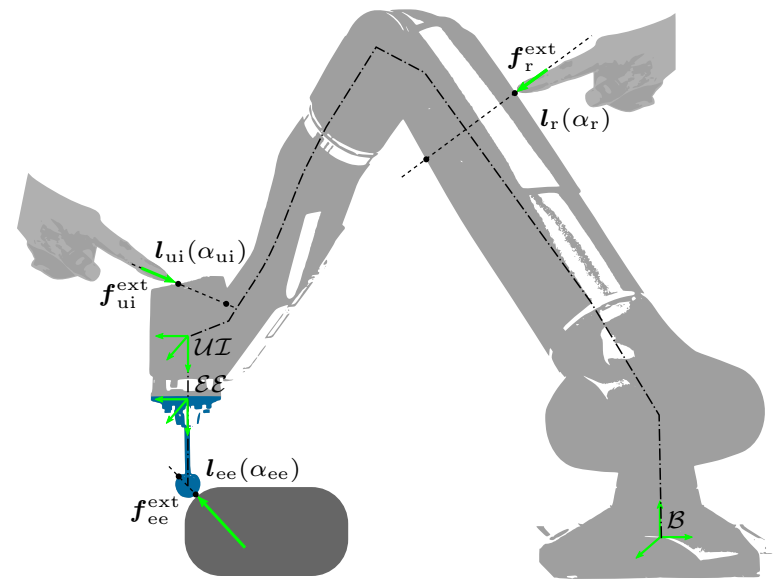

Fig. 3. The contact location is defined by the intersection between the line of force action and the robot geometry. Thanks to the sensing redundancy and the extended observer the multi-contact localization is possible.

of readability. Assuming an accurate model of the dynamics, the residual dynamics can be expressed by

$$
\dot{\overline{\boldsymbol{r}}}=\overline{\boldsymbol{K}}_{\mathrm{o}}\left(\overline{\boldsymbol{\tau}}^{\mathrm{ext}}-\overline{\boldsymbol{r}}\right),
$$

describing the relation between the extended residual and $\overline{\boldsymbol{\tau}}^{\text {ext }}$. It can be regarded as a stable, linear, decoupled, firstorder filter/estimation of the external generalized forces $\bar{\tau}^{\text {ext }}$. The observer dynamics can be expressed as

$$
\overline{\boldsymbol{r}}=\frac{\mathbf{1}}{\left(\mathbf{1} / \overline{\boldsymbol{K}}_{\mathrm{o}}\right) \boldsymbol{s}+\mathbf{1}} \overline{\boldsymbol{\tau}}^{\mathrm{ext}}, \quad \bar{r}_{i}=\frac{1}{\left(1 / \bar{K}_{\mathrm{o}, i}\right) s+1} \bar{\tau}_{i}^{\mathrm{ext}}
$$

in the Laplace domain, and the component-wise formulation for $i=1 \ldots \bar{n}$ is given in (17), which emphasizes the full decoupling of the estimated quantities. Here, the inversion of the inertia matrix is not necessary to estimate the external forces from the additional force/torque signals. Thus, a fully decoupled estimation for the external forces and wrenches is achieved. In addition, the need for joint acceleration estimates is eliminated. In this way, the sensing redundancy has been fully included in the momentum-based external force observer framework, which provides a pure external wrench estimate associated with the additional sensors.

Probably the most common disturbances exerted on the robot are due to collisions or (un)intended interactions. In general, contacts might occur in multiple locations along the structure. The literature usually considers the case of a single contact only [9], [19]. However, multiple contacts represent realistic scenarios in many practical cases. One example is human-robot-cooperation in industry where a human touches/guides the robot to perform a collaborative task. In the following, the contact location and its intensity (magnitude) are computed for single and multiple contact scenarios. The extended observer provides an estimate for the external forces in the task space already, making them configuration-independent and singularity-free.

In the SARA system, we assume specific places of interest to isolate the external forces/wrenches. The external forces in these locations are mapped to the robot base through the adjoint of the frame transformation to unify its representation. As the base sensor detects and measures the entire interaction forces and exchanges them with the ground, it perceives the total external forces collected at the base with additional torque component due to the change in lever arm. Therefore, the resultant external wrench applied along the structure ${ }_{\mathcal{B}} \tau_{\mathrm{r}}^{\text {ext }} \in \mathbb{R}^{6}$ defined in the robot base frame $\mathcal{B}$ is

$$
{ }_{\mathcal{B}} \tau_{\mathrm{r}}^{\text {ext }}=\boldsymbol{\tau}_{\mathrm{b}}^{\text {ext }}-\operatorname{Ad}_{g_{\mathcal{B}}^{\mathcal{U}}}^{T} \tau_{\mathrm{ui}}^{\text {ext }}-\operatorname{Ad}_{g_{\mathcal{B}}}^{T} \tau_{\mathrm{ee}}^{\text {ext }}
$$

where $\left.{ }_{\mathcal{B}} \boldsymbol{\tau}_{\mathrm{r}}^{\text {ext }}={ }_{\mathcal{B}} \boldsymbol{f}_{\mathrm{r}}^{\operatorname{ext} T}{ }_{\mathcal{B}} \boldsymbol{m}_{\mathrm{r}}^{\text {ext } T}\right]^{T}, \operatorname{Ad}_{g_{\mathcal{B}}^{\mathcal{I}}}$ is the adjoint of the transformation [34] from the base frame $\mathcal{B}$ to the user interface frame $\mathcal{U} \mathcal{I}$, and $\operatorname{Ad}_{g_{\mathcal{B}}^{\mathcal{E}}}$ between the two frames $\mathcal{B}$ and $\mathcal{E} \mathcal{E}$. Also, ${ }_{\mathcal{B}} \boldsymbol{f}_{\mathrm{r}}^{\text {ext }} \in \mathbb{R}^{3}$ and ${ }_{\mathcal{B}} \boldsymbol{m}_{\mathrm{r}}^{\text {ext }} \in \mathbb{R}^{3}$ represent the force and torque components, respectively, which are exerted on the structure and expressed in the base frame $\mathcal{B}$. In (18) we isolated the external forces in the other places of interaction (ui, ee). The contact location can be found/determined under the assumption that the local torque at the contact location equals zero, which is the typical case for impulsive collision and pushing. However, this assumption also applies to many situations in practice. The contact point along the structure can be extracted using the obtained external wrench from (18). Accordingly, the vector from $\mathcal{B}$ to the line of force action can be defined by the pseudoinverse solution as

$$
\boldsymbol{x}_{\mathrm{r}}=-\boldsymbol{S}\left({ }_{\mathcal{B}} \boldsymbol{f}_{\mathrm{r}}^{\mathrm{ext}}\right){ }^{\#}{ }_{\mathcal{B}} \boldsymbol{m}_{\mathrm{r}}^{\mathrm{ext}},
$$

where $S(\cdot)$ is the skew-symmetric matrix operator for the vector product. As the external force is given in $\mathcal{B}$, no further isolation for the contact link is required based on the residual components. The correct contact location can be found under the assumption of complete knowledge of the geometry and surfaces of the links. The contact point can be found by intersecting the line of force action with the geometric model. The line of force action through $\boldsymbol{x}_{\mathrm{r}}$ can be expressed as

$$
\boldsymbol{l}_{\mathrm{r}}(\alpha)=\boldsymbol{x}_{\mathrm{r}}+\alpha\left(\boldsymbol{B}_{\mathrm{r}}^{\mathrm{ext}} /\left\|_{\mathcal{B}} \boldsymbol{f}_{\mathrm{r}}^{\mathrm{ext}}\right\|\right),
$$

where $\alpha \in \mathbb{R}$ is a varying scalar. In principle, all points in the line of force action are valid candidates. Nevertheless, given the knowledge of the surface $\mathcal{S}_{i_{c}}$ of the contact link, this line intersects with $\mathcal{S}_{i_{c}}$ in two locations. The first location represents a pushing force to the robot (link) surface and the second one corresponds to a pulling force out of the surface. As no adhesive forces are assumed, one is generally interested in the first intersection. According to this choice, the smaller value of the scalar $\alpha=\alpha_{\mathrm{r}}$ can be found which corresponds to a pushing force to compute the correct contact location on the structure $l_{\mathrm{r}}\left(\alpha_{\mathrm{r}}\right)$ as shown in Fig. 3. Now as the external contact force $\mathcal{B}_{\mathrm{r}}^{\text {ext }}$ is expressed in the base frame, the magnitude of the contact force along the structure is directly $\mathcal{B}_{\mathrm{r}}^{\text {ext }}$ which is applied at the point $\boldsymbol{l}_{\mathrm{r}}\left(\alpha_{\mathrm{r}}\right)$. Similarly, the external wrench at the end-effector level could be also mapped to $\mathcal{B}$ as

$$
\mathcal{B}_{\mathrm{ee}}^{\mathrm{ext}}=\operatorname{Ad}_{g_{\mathcal{B}}^{\mathcal{E}}}^{T} \tau_{\mathrm{ee}}^{\mathrm{ext}}
$$

where ${ }_{\mathcal{B}} \boldsymbol{\tau}_{\mathrm{ee}}^{\mathrm{ext}}=\left[{ }_{\mathcal{B}} \boldsymbol{f}_{\mathrm{ee}}^{\mathrm{ext} T}{ }_{\mathcal{B}} \boldsymbol{m}_{\mathrm{ee}}^{\mathrm{ext} T}\right]^{T}$. Analogous to (19), the minimum distance from $\mathcal{B}$ to the line of force action can be defined by the pseudoinverse solution as

$$
\boldsymbol{x}_{\mathrm{ee}}=-\boldsymbol{S}\left({ }_{\mathcal{B}} \boldsymbol{f}_{\mathrm{ee}}^{\mathrm{ext}}\right)^{\#}{ }_{\mathcal{B}} \boldsymbol{m}_{\mathrm{ee}}^{\mathrm{ext}}
$$


and with reference to (20) the end-effector contact point $\boldsymbol{l}_{\text {ee }}\left(\alpha_{\mathrm{ee}}\right)$ can be found, which is specified by the scalar $\alpha_{\text {ee }}$. This process can be repeated multiple times to cover all available/redundant sensors and the areas of interest; here for example, to find the contact on the user interface part/place $l_{\text {ui }}\left(\alpha_{\text {ui }}\right)$. Conventionally while trying to localize even a single point (i.e., when the collision occurs at the link $i_{c}$ ), the contact Jacobian $\boldsymbol{J}_{c}(\boldsymbol{q})$ includes $n-i_{c}$ zerocolumns. Because it does not include additional sensors in the scheme. Here the restriction/limitation on the number of joints affected by the collision does not arise, which is $i_{c}<6$ for the full external wrench in the conventional approach (state-of-the-art approaches) [19]. In fact, there is the ability to detect and localize point collision, which is near the robot base, and even below the first joint of the robot; this is due to the placement of the sensors in the considered SARA robot. The additional sensors can be integrated into the observer dynamics, and they are treated as internal sensing devices and as one part of the robot. Therefore, the extended observer avoids the above-mentioned drawbacks. The proposed approach enables the implementation/realization of more advanced collision reaction strategies. It provides rich information about the level of collision (at the end-effector or/and on the structure). For instance, if $\left|\mathcal{B} \tau_{\mathrm{r}}^{\text {ext }}\right|>\epsilon_{1 . . n_{b}}$, a collision can be detected and identified on the structure; in this case, a specific logic/heuristic can be developed for a collision reaction strategy depending on the collision level. The parameter $\epsilon \in \mathbb{R}^{\bar{n}}$ represents a threshold vector to enhance the robustness of the collision detection against unmodeled dynamics, and it eliminates false positives [7].

Different applications and scenarios could take advantage of estimating the end-effector external forces and the intentional user interactions, such as teaching the robot a task that includes forces by demonstration. This is because all user interactions could be isolated from the task external forces as they are estimated by two individual residual wrenches. The assumption of the absence of external torques does not generally hold at all of the contacts. In fact, any place/point could include forces and torques (complete wrench) as they are isolated from each other, and they are not influenced by other contact forces. For instance, the robot may perform a task that requires exerting torques in the environment while localizing the contact collision at the structure. In this case, a full estimate of the end-effector external wrench can be obtained but without providing the contact location. In order to simultaneously detect and isolate more than $n$ external forces in an $n$-DOF mechanical system, the additional DOF that are collocated with the redundant sensing have to be also uncoupled. Using the estimated generalized acceleration for compensating the dynamical effects for the additional sensing devices results in a coupled system. Thus simultaneous detection and isolation are not possible. The extended observer is not limited to detecting and isolating $n$ external forces but can simultaneously estimate more than the number of active DOF, namely, $\bar{n}$, including the additional dimensions of the force-torque sensors. Due to the introduction of additional virtual coordinates (joints), the dimension
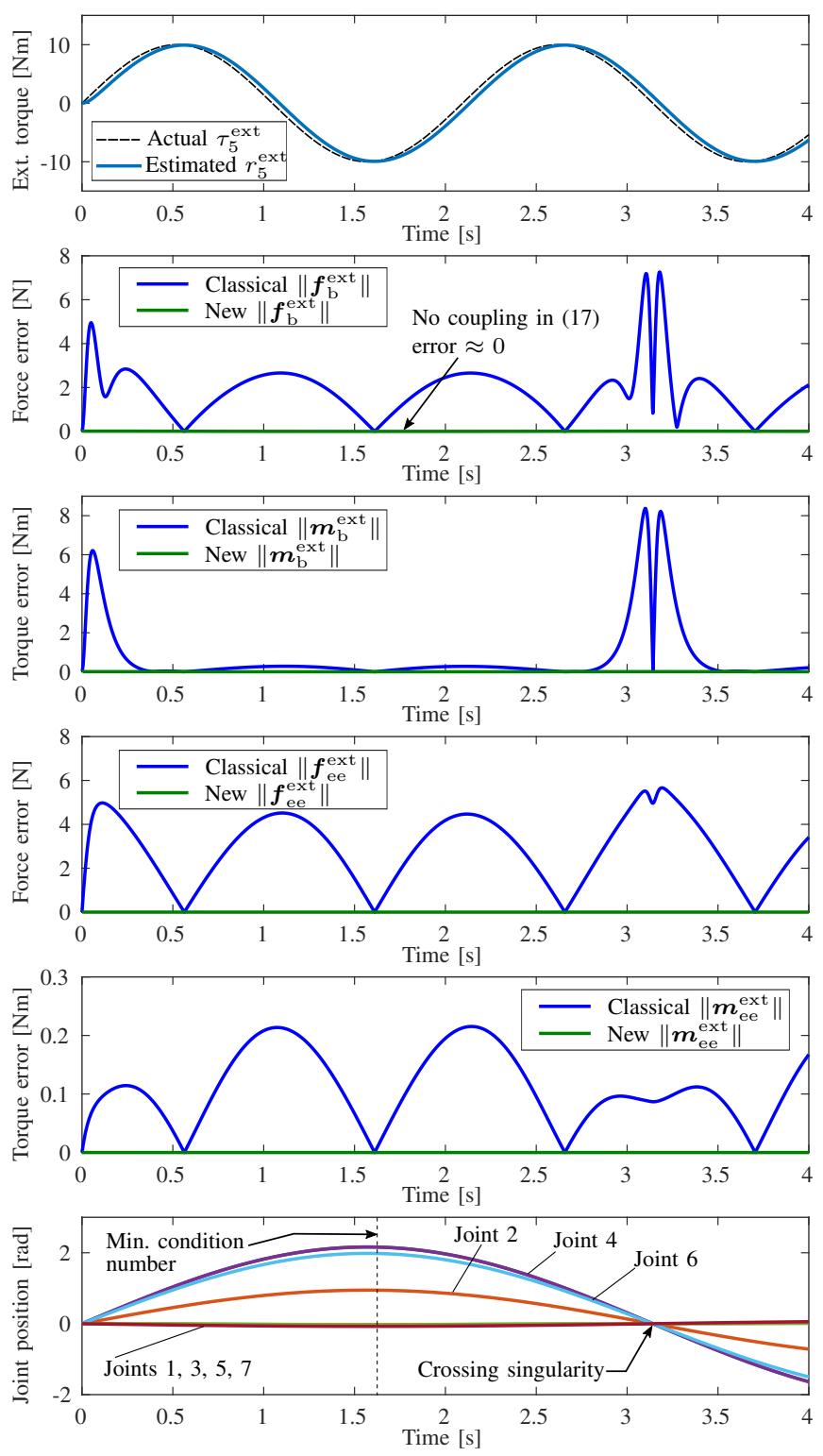

Fig. 4. Dynamical uncoupling behavior with the additional sensors in case of continuously active single DOF disturbance is shown. Theoretically, the proposed method provides a zero coupling effect in the estimated external forces and torques of the considered six-DOF sensors.

of the dynamic equations also increased, which demands more computational power to provide a real-time estimate. However, as no matrix inversion is required, that should not pose a problematic limiting factor in most practical setups. In fact, at the SARA system, the new observer runs in real-time at a rate of $8 \mathrm{kHz}$. Additionally, the observer is entirely model-based and thus requires an explicit and precise dynamical model of the robotic system.

\section{Simulations And Experiments}

In this section, the proposed extension of the momentumbased observer is validated in simulations and experiments. The experiments are conducted on the DLR lightweight robot SARA [28]. In SARA, all force-torque sensors are fully integrated within the mechanical structure. These sensors are of straingauge-based type with optimized design to increase 

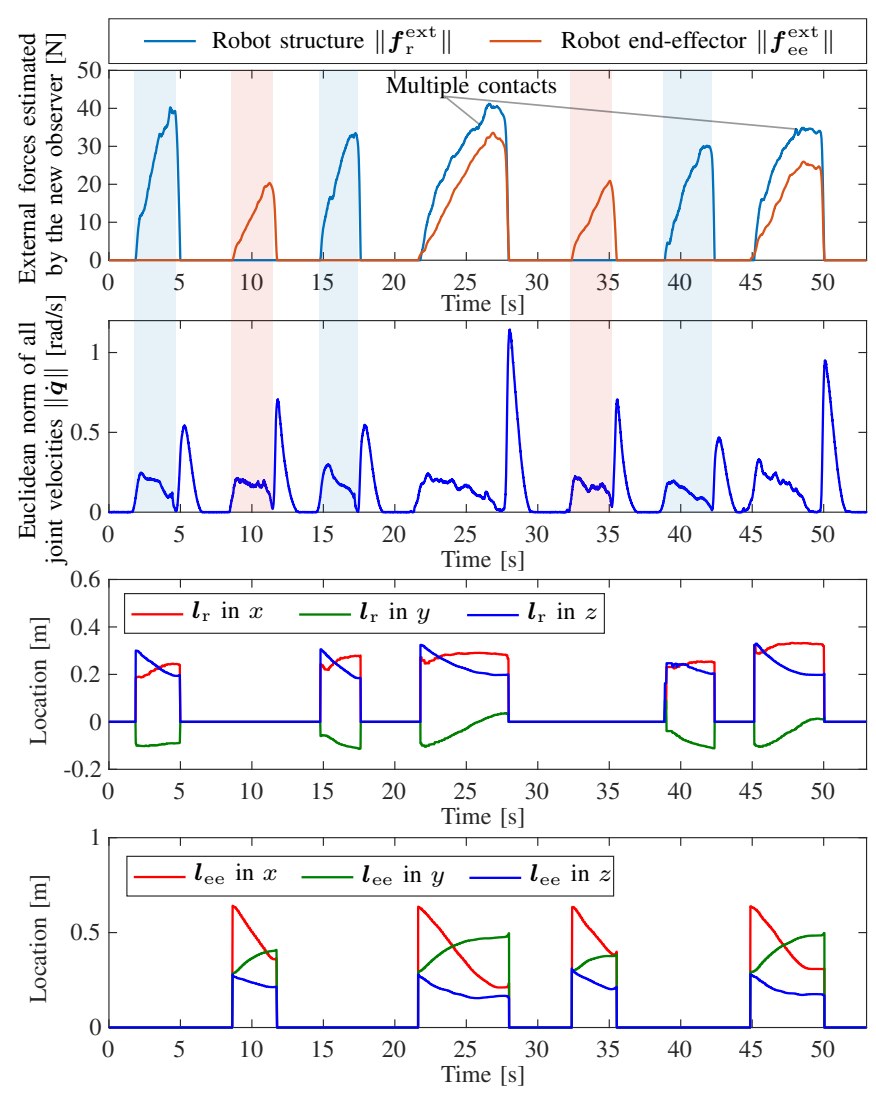

Fig. 5. Experimental results for the dynamic identification and localization of multiple contacts. The locations of the contacts on the robot structure $\boldsymbol{l}_{\mathrm{r}}$ and at the end-effector level $\boldsymbol{l}_{\mathrm{ee}}$ are illustrated.

the sensor resolution. The robot has two 6-DOF force-torque sensors, one in the base and another one in the robot wrist. Also, the system features additional sensors in the revolute joints in between. ${ }^{1}$ Consequently, the system is equipped with instrumentation providing $6+4+6=16$ signals.

In the following simulation on SARA, a sinusoidal disturbance with amplitude of $10 \mathrm{Nm}$ is applied to joint five as external torque, as shown in Fig. 4 (top diagram) while performing a joint-level trajectory (bottom diagram). The trajectory includes the configuration with minimum condition number as well as the crossing of a singularity w. r. t. the full Cartesian space of the end-effector. The external forces and torques at the end-effector and the robot base are computed using the classical approach in [9] via the estimated generalized accelerations (6). Subsequently, $\ddot{\hat{\boldsymbol{q}}}$ from (6) is used to feed a parallel dynamical computation in order to obtain the external wrench perceived by the force-torque sensors. That strategy is basically equivalent to the use of NewtonEuler computations combined with the estimated joint accelerations, see [9], [16]. Notably, as both approaches employ the generalized momentum on joint level, the proposed extended observer and the momentum-based disturbance observer [7] are equivalent to estimate the external joint torques. However, the estimated external Cartesian wrenches

\footnotetext{
${ }^{1}$ The base and the wrist sensor are also used in the local control of the adjacent joints. Moreover, a torque sensor is added for the user interface.
}

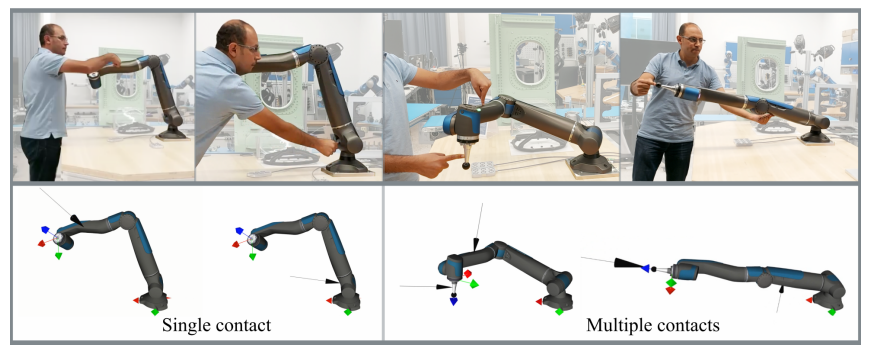

Fig. 6. Experimental identification of single and multiple contacts.

differ in both approaches. In Fig. 4, one can clearly see the dynamic coupling in the classical approach represented by force and torque errors. The joint-acceleration estimate $\ddot{\hat{\boldsymbol{q}}}$ directly relies on the estimated external torques through the residual. As the residual is a first-order filtered version of the external torques, an error is inherited during transients. Consequently, this error in $\ddot{\hat{\boldsymbol{q}}}$ is nonlinearly coupled through $\boldsymbol{M}(\boldsymbol{q})^{-1}$ in (6). Also, one can clearly see that the errors shown in Fig. 4 are configuration-dependent. In contrast, the extended observer framework is able to handle the challenge of the dynamic coupling, provides fully decoupled external force and torque estimates, and it eliminates the need for acceleration estimates.

In the following experiment, the identification of multiple contacts (forces, locations) is demonstrated. The robot is operated in joint-level impedance control mode to keep a specific configuration compliantly. In Fig. 5 (top) one can observe the external force as a result of the physical interaction. After releasing the external contacts, the initial configuration is approached again. This can be seen in $\|\dot{\boldsymbol{q}}\|$ in Fig. 5, where high-velocity phases occur after the contact. During these phases, a full decoupling is achieved, confirming the theoretical results from Sec. III. Additionally, the multiple contacts are estimated independently/separately along the structure and at the end-effector level. As mentioned in Section III, to increase the robustness of the collision detection, a threshold is used, no contact location is assumed below the defined force threshold. The results of multiple contact scenarios are demonstrated in Fig. 6 and in the video attachment, where the identification of the contact point near the robot base as well as in the singular direction is highlighted.

\section{Conclusions}

A unified momentum-based framework to estimate external forces was presented. In addition to joint-level residuals, the approach provides uncoupled force-torque estimates in the Cartesian space, making these estimates independent of the joint configuration and not affected by singularities. The observer formulation allows the straightforward integration of additional sensory devices in arbitrary locations of the kinematic chain, such as in the base or at the end-effector. The framework eliminates the need for acceleration measurements or estimates. Moreover, multiple simultaneous external contacts can be robustly identified and localized in real time. Dynamical simulations and experiments on the DLR robot SARA with force-torque sensing redundancy confirm the theoretical claims. 


\section{REFERENCES}

[1] S. Haddadin, A. Albu-Schäffer, A. De Luca, and G. Hirzinger, "Collision Detection and Reaction: A Contribution to Safe Physical HumanRobot Interaction," in Proc. of the 2008 IEEE/RSJ International Conference on Intelligent Robots and Systems, September 2008, pp. 3356-3363.

[2] S. Morinaga and K. Kosuge, "Collision detection system for manipulator based on adaptive impedance control law," in 2003 IEEE International Conference on Robotics and Automation, vol. 1. IEEE, 2003, pp. 1080-1085.

[3] K. Ohnishi, M. Shibata, and T. Murakami, "Motion Control for Advanced Mechatronics," IEEE/ASME Transactions on Mechatronics, vol. 1, no. 1, pp. 56-67, March 1996.

[4] S. Haddadin, Towards Safe Robots: Approaching Asimov's 1st Law, ser. Springer Tracts in Advanced Robotics. Springer-Verlag Berlin Heidelberg, 2014, vol. 90.

[5] C. Lee, J. Lee, J. Malzahn, N. Tsagarakis, and S. Oh, "A twostaged residual for resilient external torque estimation with series elastic actuators," in 2017 IEEE-RAS 17th International Conference on Humanoid Robotics (Humanoids). IEEE, 2017, pp. 817-823.

[6] G. Garofalo, N. Mansfeld, J. Jankowski, and C. Ott, "Sliding mode momentum observers for estimation of external torques and joint acceleration," in 2019 International Conference on Robotics and Automation (ICRA). IEEE, 2019, pp. 6117-6123.

[7] A. De Luca, A. Albu-Schäffer, S. Haddadin, and G. Hirzinger, "Collision Detection and Safe Reaction with the DLR-III Lightweight Manipulator Arm," in Proc. of the 2006 IEEE/RSJ International Conference on Intelligent Robots and Systems, October 2006, pp. $1623-1630$.

[8] E. Magrini, F. Flacco, and A. De Luca, "Estimation of contact forces using a virtual force sensor," in 2014 IEEE/RSJ International Conference on Intelligent Robots and Systems. IEEE, 2014, pp. 21262133.

[9] G. Buondonno and A. De Luca, "Combining real and virtual sensors for measuring interaction forces and moments acting on a robot," in 2016 IEEE/RSJ International Conference on Intelligent Robots and Systems (IROS). IEEE, 2016, pp. 794-800.

[10] S. Lu, J. H. Chung, and S. A. Velinsky, "Human-robot collision detection and identification based on wrist and base force/torque sensors," in Proceedings of the 2005 IEEE international Conference on Robotics and Automation. IEEE, 2005, pp. 3796-3801.

[11] F. Lange, W. Bertleff, and M. Suppa, "Force and trajectory control of industrial robots in stiff contact," in 2013 IEEE International Conference on Robotics and Automation. IEEE, 2013, pp. 29272934.

[12] G. Liu, K. Iagnemma, S. Dubowsky, and G. Morel, "A base force/torque sensor approach to robot manipulator inertial parameter estimation," in Proceedings. 1998 IEEE International Conference on Robotics and Automation (Cat. No. 98CH36146), vol. 4. IEEE, 1998, pp. 3316-3321.

[13] C. Ott and Y. Nakamura, "Base force/torque sensing for position based cartesian impedance control," in 2009 IEEE/RSJ International Conference on Intelligent Robots and Systems. IEEE, 2009, pp. 32443250 .

[14] K. Kosuge, T. Oosumi, Y. Hirata, H. Asama, H. Kaetsu, and K. Kawabata, "Handling of a single object by multiple autonomous mobile robots in coordination with body force sensor," in Proceedings. 1998 IEEE/RSJ International Conference on Intelligent Robots and Systems. Innovations in Theory, Practice and Applications (Cat. No. 98CH36190), vol. 3. IEEE, 1998, pp. 1419-1424.

[15] G. Morel, K. Iagnemma, and S. Dubowsky, "The precise control of manipulators with high joint-friction using base force/torque sensing," Automatica, vol. 36, no. 7, pp. 931-941, 2000

[16] M. Fumagalli, S. Ivaldi, M. Randazzo, L. Natale, G. Metta, G. Sandini, and F. Nori, "Force feedback exploiting tactile and proximal force/torque sensing," Autonomous Robots, vol. 33, no. 4, pp. 381398,2012
[17] S. Traversaro, A. Del Prete, S. Ivaldi, and F. Nori, "Inertial parameters identification and joint torques estimation with proximal force/torque sensing," in 2015 IEEE International Conference on Robotics and Automation (ICRA). IEEE, 2015, pp. 2105-2110.

[18] J. Vorndamme, M. Schappler, and S. Haddadin, "Collision detection, isolation and identification for humanoids," in 2017 IEEE International Conference on Robotics and Automation (ICRA). IEEE, 2017, pp. 4754-4761.

[19] S. Haddadin, A. De Luca, and A. Albu-Schäffer, "Robot collisions: A survey on detection, isolation, and identification," IEEE Transactions on Robotics, vol. 33, no. 6, pp. 1292-1312, 2017.

[20] A. Zwiener, C. Geckeler, and A. Zell, "Contact point localization for articulated manipulators with proprioceptive sensors and machine learning," in 2018 IEEE International Conference on Robotics and Automation (ICRA). IEEE, 2018, pp. 323-329.

[21] L. Manuelli and R. Tedrake, "Localizing external contact using proprioceptive sensors: The contact particle filter," in 2016 IEEE/RSJ International Conference on Intelligent Robots and Systems (IROS). IEEE, 2016, pp. 5062-5069.

[22] S. Takakura, T. Murakami, and K. Ohnishi, "An approach to collision detection and recovery motion in industrial robot," in 15th Annual Conference of IEEE Industrial Electronics Society. IEEE, 1989, pp. 421-426.

[23] A. Petrovskaya, J. Park, and O. Khatib, "Probabilistic estimation of whole body contacts for multi-contact robot control," in Proceedings 2007 IEEE International Conference on Robotics and Automation. IEEE, 2007, pp. 568-573.

[24] M. Iskandar and S. Wolf, "Dynamic friction model with thermal and load dependency: modeling, compensation, and external force estimation," in 2019 IEEE International Conference on Robotics and Automation (ICRA). IEEE, May 2019, pp. 7367-7373.

[25] S. Wolf and M. Iskandar, "Extending a dynamic friction model with nonlinear viscous and thermal dependency for a motor and harmonic drive gear," in 2018 IEEE International Conference on Robotics and Automation (ICRA). IEEE, May 2018, pp. 783-790.

[26] L. Le Tien, A. Albu-Schäffer, A. De Luca, and G. Hirzinger, "Friction observer and compensation for control of robots with joint torque measurement," in 2008 IEEE/RSJ International Conference on Intelligent Robots and Systems. IEEE, 2008, pp. 3789-3795.

[27] M. J. Kim, F. Beck, C. Ott, and A. Albu-Schäffer, "Model-free friction observers for flexible joint robots with torque measurements," IEEE Transactions on Robotics, vol. 35, no. 6, pp. 1508-1515, 2019.

[28] M. Iskandar, C. Ott, O. Eiberger, M. Keppler, A. Albu-Schäffer, and A. Dietrich, "Joint-level control of the dlr lightweight robot sara," in Proc. of the 2020 IEEE/RSJ International Conference on Intelligent Robots and Systems. IEEE, 2020, pp. 8903-8910.

[29] A. Dietrich and C. Ott, "Hierarchical Impedance-Based Tracking Control of Kinematically Redundant Robots," IEEE Transactions on Robotics, vol. 36, no. 1, pp. 204-221, February 2020.

[30] A. Dietrich, T. Wimböck, A. Albu-Schäffer, and G. Hirzinger, "Reactive Whole-Body Control: Dynamic Mobile Manipulation Using a Large Number of Actuated Degrees of Freedom," IEEE Robotics \& Automation Magazine, vol. 19, no. 2, pp. 20-33, June 2012.

[31] M. Iskandar, G. Quere, A. Hagengruber, A. Dietrich, and J. Vogel, "Employing whole-body control in assistive robotics," in IEEE International Conference on Intelligent Robots and Systems, November 2019, pp. 5643-5650

[32] J. Vogel, S. Haddadin, B. Jarosiewicz, J. D. Simeral, D. Bacher, L. R. Hochberg, J. P. Donoghue, and P. van der Smagt, "An assistive decision-and-control architecture for force-sensitive hand-arm systems driven by human-machine interfaces," The International Journal of Robotics Research, vol. 34, no. 6, pp. 763-780, 2015

[33] A. Dietrich, C. Ott, and A. Albu-Schäffer, "Multi-Objective Compliance Control of Redundant Manipulators: Hierarchy, Control, and Stability," in Proc. of the 2013 IEEE/RSJ International Conference on Intelligent Robots and Systems, November 2013, pp. 3043-3050.

[34] R. M. Murray, Z. Li, and S. S. Sastry, A Mathematical Introduction to Robotic Manipulation. CRC Press, 1994. 